\title{
Brain abscesses in children: an Italian multicentre study
}

\author{
I. RAFFALDI ${ }^{1}$, S. GARAZZINO ${ }^{1}$, G. CASTELLI GATTINARA ${ }^{2}$, R. LIPRERI $^{3}$, \\ L. LANCELLA ${ }^{4}$, S. ESPOSITO ${ }^{5}$, A. M. GIANNINI ${ }^{6}$, C. MONTAGNANI ${ }^{7}$, \\ G. L. MARSEGLIA ${ }^{8}$, C. PIGNATA ${ }^{9}$, F. BERNARDI $^{10}$, P.-A. TOVO $^{1}$ AND \\ SITIP BRAIN ABSCESSES REGISTRY† \\ ${ }^{1}$ University of Turin, Regina Margherita Children's Hospital, Turin, Italy \\ ${ }^{2}$ Bambino Gesù Children Hospital, Rome, Italy \\ ${ }^{3}$ TB Reference Centre/Villa Marelli Institute, Niguarda Hospital, Milan, Italy \\ ${ }^{4}$ IRCCS Bambino Gesù Hospital, Rome, Italy \\ ${ }^{5}$ University of Milan, Fondazione IRCCS Ca' Granda Ospedale Maggiore Policlinico, Milan, Italy \\ ${ }^{6}$ Giovanni XXIII Hospital, Bari, Italy \\ ${ }^{7}$ University of Florence, Meyer Hospital, Florence, Italy \\ ${ }^{8}$ University of Pavia, IRCCS Policlinico 'S. Matteo' Foundation, Pavia, Italy \\ ${ }^{9}$ University of Naples 'Federico II', Naples, Italy \\ ${ }^{10}$ University of Bologna, S. Orsola-Malpighi Hospital, Bologna, Italy
}

Received 31 October 2016; Final revision 19 June 2017; Accepted 30 June 2017; first published online 3 August 2017

\section{SUMMARY}

Brain abscess is uncommon in paediatric population, but of clinical importance because of significant long-term morbidity and mortality. In this multicentre study, promoted by the Italian Society for Paediatric Infectious Diseases, we retrospectively collected patients aged 0-18 years, with a diagnosis of 'brain abscess'. Seventy-nine children were included; the median age was 8.75 years. As predisposing factor, 44 children had preceding infections. The Gram-positive cocci were mostly isolated (27 cases). Sixty $(76 \%)$ children underwent a surgical intervention. Intravenous antibiotic therapy was administered in all patients, then switched to oral treatment. Clinical sequelae were recorded in $31(39 \cdot 2 \%)$ children. Twenty-one of them had a single sequela, of which, the most represented, was epilepsy in nine of them. This study focus the attention on the need to have standardized national guidelines or adequate recommendations on type and duration of antibiotic treatment.

Key words: Anaerobic bacteria, antibiotics, bacterial infections, central nervous system infections, emerging infections.

\footnotetext{
* Author for correspondence: I. Raffaldi, University of Turin, Regina Margherita Children's Hospital, Piazza Polonia 94 10126, Turin, Italy.

(Email: ire_raffaldi@yahoo.it)

† SITIP Brain Abscesses Registry: C. Scolfaro, P. P. Gaglini, G. Pattarino, C. Tagliabue, L. Galli, S. Caimmi, F. Cipriani, F. Nicolini, A. Berardi, A. Lo Vecchio, G. Ausanio, N. Rossi and S. Norbedo.
}

\section{INTRODUCTION}

Brain abscess (BA) is an infection that involves the brain parenchyma starting as a localized area of cerebritis, which evolves into a collection of encapsulated purulent material [1]. It is uncommon in paediatric population, but of clinical importance because of significant long-term morbidity and mortality [2-4]. BAs in children were traditionally associated with 
congenital heart diseases and infections of the adjacent anatomic sites; the infection can spread haematogenously or by contiguity [2,3]. Probably due to improvement in imaging and neurosurgical techniques and the introduction of new antibiotic regimens, the mortality has decreased, from $30 \%$ to $60 \%$ in the late 1970 s, to $<10 \%$ in recent years [5].

The clinical onset of BA may be sneaky and it depends on the age of the patient and on the size, location and number of lesions [6]. The classic triad of fever, headache and focal neurological signs was seen in $9-28 \%$ of the paediatric cases $[6,7]$.

The most often isolated pathogens of BAs belong to Streptococcus spp., Staphylococcus spp. and Gramnegative bacteria [8,9]. Among these, Streptococcus spp. are markedly common in cases with paranasal sinusitis, otitis and periorbital cellulitis, which are, according to literature, more frequent predisposing conditions for intracranial abscess in children [3]. Surgical treatment, associated with empirical broadspectrum antimicrobial therapy, is widely recommended $[1,2,5]$.

To our knowledge, there are only few surveys in the European setting concerning the management of BAs $[2,5,6]$. Other published studies generally analyze both adults and children without providing a subset analysis for the paediatric population $[9,10]$.

This is a retrospective, multicentre, observational study, promoted by the Italian Society for Paediatric Infectious Diseases (SITIP).

The aim of the survey was to analyze the clinical presentation, risk factors, bacteriological isolates, treatment and outcomes of children and adolescents with a diagnosis of BA in Italy. Moreover, we have reviewed and documented the temporal changes in the predisposing factors and causative pathogens of intracranial abscesses.

\section{MATERIALS AND METHODS}

All the institutions included in the network of the SITIP received an invitation to participate in this retrospective study; 15 paediatric centres agreed to participate and reported at least one case of BA.

We have included children and adolescents aged 018 years, admitted from 1 January 1998 to 31 May 2013 with a diagnosis of 'BA'.

According to more recent definitions, we have considered BA a lesion located in the cerebrum, midbrain or cerebellum, with characteristic features at computed tomography (CT) or magnetic resonance imaging (MRI) and/or evidence of pus during surgery $[6,7]$.

The World Health Organization International Classification of Disease (ICD) version 10, code G06.0 for intracranial abscess, was used. Patients with subdural and epidural empyemas, fungal, parasitic, protozoa or tubercular abscesses were excluded.

Medical records have been carefully reviewed to collect data on patient demographics (age and gender), predisposing factors, signs and symptoms at presentation, site and size of BAs, microbiological and laboratory findings (peripheral white blood count - WBC - and C reactive protein - CRP), radiological imaging and treatment regimen. Data concerning outcomes, sequelae (epilepsy, focal motor deficits, visual impairment, hydrocephalous and speaking, hearing or learning disorders) and infection recurrence were evaluated.

To analyze the temporal changes in predisposing factors and responsible microorganisms, we have split the study period in two: 1998-2005 and 2006-2013.

Statistical analysis was performed using Ministat 2.1 and OpenEpi. The differences between groups were analyzed using the $t$ test or Mann-Whitney test for continuous data, or $\chi^{2}$ test or the Fisher exact test for categorical data, as appropriate. All tests were two sided and the significance was set at $P \leqslant 0 \cdot 05$.

\section{RESULTS}

Ninety-four potential cases were identified, but 15 were excluded on the basis of the exclusion criteria (seven post-meningitis empyemas and eight fungal abscesses). Therefore, 79 children were included in the study (male-to-female ratio was 1.8); the median age was 8.75 years (range IQR $2.46-12.33$ years). It might be thought that the majority of patients $(55 \cdot 6 \%)$ are actually children aged $<10$ years. Males were not significantly older than girls (median $9 \cdot 4 \mathrm{vs}$. $4 \cdot 4$ years, $P=0 \cdot 10)$.

\section{Predisposing factors}

The predisposing factors were identified in $68(86 \%)$ subjects: $44(55 \cdot 7 \%)$ had preceding infections (16 rhinosinusitis - 14 frontal and two involving ethmoidal sinus - seven acute otitis, seven acute mastoiditis, six sepsis, three orbital cellulitis, three dental abscess, one endocarditis and one parapharyngeal abscess), seven $(8.9 \%)$ underwent a previous neurosurgical procedures, six $(7 \cdot 6 \%)$ had a congenital heart disease, four 
$(5 \%)$ had a head trauma, four $(5 \%)$ were immunocompromised (two had a congenital form and two had an acquired immunosuppression) and finally three (3.8\%) had brain malformations. No predisposing factors were identified in $11(14 \%)$ of subjects.

Eight $(42 \%)$ of 19 cases reported in the first period had a preceding infection, compared with $36(60 \%)$ of 60 cases reported during the second period $(P=0 \cdot 19)$; whereas the congenital heart diseases were less frequent in both periods: two $(10 \%)$ cases and four (6\%) cases, respectively, in 1998-2005 and 2006$2013(P=0 \cdot 69)$.

\section{Clinical features}

In $70(88.6 \%)$ children, signs and symptoms at admission have been documented (see Table 1). The median duration of signs and symptoms before the diagnosis of BA was 7 days (range IQR 2-13.5 days).

Because of the suspected infection, $36(45 \cdot 6 \%)$ children had previously received an antibiotic therapy, particularly $\beta$ lactams $(n=23)$, for a mean duration of $8 \cdot 7 \pm 7 \cdot 3$ days (range $2-37$ days).

\section{Laboratory findings}

In $72(91 \cdot 1 \%)$ patients, laboratory data on admission were available. The median WBC count was 14210 cells $/ \mathrm{mm}^{3}$ (range IQR $10140-18700$ cells $/ \mathrm{mm}^{3}$ ); in $56(77 \cdot 7 \%)$ children the WBC count was $>10000 /$ $\mathrm{mm}^{3}$; whereas three $(4 \cdot 1 \%)$ had leucopaenia with $<5000$ cells $/ \mathrm{mm}^{3}$. The CRP was increased $(\geqslant 5 \mathrm{mg} / \mathrm{l})$ in $52(72 \%)$ patients, with a median level of $10 \cdot 1$ $\mathrm{mg} / \mathrm{l}$ (range IQR $3 \cdot 5-18 \cdot 4 \mathrm{mg} / \mathrm{l}$ ).

Blood cultures were performed in 44 out of 79 $(55.7 \%)$ children, but they were positive only in 10 of the 44 cases (positive rate $=22 \cdot 7 \%$ ). In these 10 positive blood cultures, the same microorganism has been isolated also in brain pus.

The lumbar puncture was performed in $25(31 \cdot 6 \%)$ children, in the initial suspicion of meningitis: the mean cell count was $4792 \cdot 2 \pm 10976 \cdot 4 / \mathrm{mm}^{3}$, the mean protein level was $212 \cdot 8 \pm 278 \cdot 2 / \mathrm{mm}^{3}$ and the glucose level was $38.9 \pm 26 \cdot 3 \mathrm{mg} / \mathrm{dl}$. The cerebrospinal fluid culture was positive in six cases (positive rate $=24 \%$ ).

\section{Radiological findings}

Neuroimaging study was available in 76 patients: 69 (90.7\%) of them had CT scan on admission; whereas, in the other seven $(9 \cdot 3 \%)$, the MRI was the first cerebral imaging.

There were $58(73 \cdot 4 \%)$ single BAs and $21(26 \cdot 6 \%)$ multiple. The location, for solitary lesions, is presented in Table 2. The mean abscess diameter was $2 \cdot 7 \pm 1 \cdot 5 \mathrm{~cm}$ (range $0 \cdot 25-5 \cdot 7 \mathrm{~cm}$ ).

\section{Management}

Sixty $(76 \%)$ children underwent a surgical intervention: $51 / 60(85 \%)$ had a drainage of the lesion, $7 / 60$ $(11 \cdot 7 \%)$ had a resection and $2 / 60(3 \cdot 3 \%)$ had other surgical approach. The time between the onset of symptoms and surgery ranged from 0 to 22 days, for those so treated.

Subjects who received surgical treatment were mainly males (39 over 60 children, $65 \%$ ) with a median age of 8.9 years (IQR $2.4-12.2$ years). The mean length of hospital stay was $43 \cdot 5 \pm 23.6$ days (range 9-140 days). They received a previous antibiotic therapy in $25 / 60$ cases $(41 \cdot 7 \%)$. A single BA was more frequent in these subjects (47 over 60 cases, 78.3\%) and the most usual location was the frontal lobe (20/60 BAs, 33.3\%), with a mean BA's diameter of $3 \pm 1.6 \mathrm{~cm}$ (range $0.5-6 \mathrm{~cm}$ ), which was significantly greater than those who did not receive surgical treatment $(P=0 \cdot 0005, \mathrm{CI}$ : confidence interval $95 \% 0 \cdot 46-0 \cdot 56)$. The mean length of antibiotic treatment, in these subjects, was $57 \cdot 8 \pm 30 \cdot 2$ days (range 15-159 days). Twenty-four of them (40\%) developed a sequela. In surgical treated patients, we recorded $6 / 60(10 \%)$ recurrence and 1/60 (1.6\%) death.

Examining separately, those who underwent resection had single BA in $6 / 7$ cases $(85.7 \%)$, with a prevalent location in the frontal lobe (three cases over seven, $42 \cdot 8 \%$ ). No recurrence occurred.

Similarly, those who received the drainage had frequently a single lesion $(40 / 51,78 \cdot 4 \%)$, located in the frontal lobe $(17 / 51 \mathrm{BAs}, 33 \cdot 3 \%)$. No correlations were found between abscess location and surgical procedures $(P=0 \cdot 68)$. Recurrence was recorded in six cases $(6 / 51,11 \cdot 8 \%)$.

Children who received only medical treatment were 12 males and seven females with a median age of $5 \cdot 6$ years (IQR 1.9-12 years). The mean duration of hospital stay, for this group, was $50 \cdot 4 \pm 19 \cdot 8$ days (range $17-98$ days). Eleven of them $(11 / 19,57 \cdot 9 \%)$ had received a previous oral antibiotic therapy. The BA was single in 11 cases over $19(57.9 \%)$, and the prevalent location was the frontal lobe (4/19 BAs, $21 \%)$. The mean BA diameter, in this group, was $1.7 \pm 0.9 \mathrm{~cm}$ 
Table 1. Signs and symptoms of brain abscess at admission

\begin{tabular}{ll}
\hline \hline & No. of patients $(\%)$ \\
\hline General signs and symptoms & \\
Fever & $53(67)$ \\
Headache & $48(60 \cdot 7)$ \\
Vomiting & $31(39 \cdot 2)$ \\
Altered level of consciousness & $28(35 \cdot 4)$ \\
Dizziness & $5(6 \cdot 3)$ \\
Increasing head circumference & $2(2 \cdot 5)$ \\
Bulging fontanel & $1(1 \cdot 2)$ \\
Neurological signs and symptoms & \\
Generalized tonic-clonic seizures & $17(21 \cdot 5)$ \\
Hemiplegia & $9(11 \cdot 3)$ \\
Expressive dysphasia & $9(11 \cdot 3)$ \\
Abnormal gait & $9(11 \cdot 3)$ \\
Partial seizures & $8(10 \cdot 1)$ \\
Cranial nerve palsy & $3(3 \cdot 7)$ \\
Status epilepticus & $2(2 \cdot 5)$ \\
Hypotonia & $1(1 \cdot 2)$ \\
Visual signs and symptoms & \\
Photophobia & $8(10 \cdot 1)$ \\
Papillo-oedema & $2(2 \cdot 5)$ \\
Ptosis & $2(2 \cdot 5)$ \\
Decreased visual acuity & $1(1 \cdot 2)$ \\
Diplopia & $1(1 \cdot 2)$ \\
\hline \hline
\end{tabular}

(range $0 \cdot 3-3 \mathrm{~cm}$ ). The mean duration of antibiotic therapy was $65 \cdot 3 \pm 21 \cdot 2$ days (range 30-119 days), not significantly longer than those who underwent surgical treatment $(P=0 \cdot 22)$. Eight children $(8 / 19$, $42 \cdot 1 \%$ ) developed a sequela; nor recurrences neither deaths were recorded in this group.

Intravenous (i.v.) antibiotic therapy was administered in all patients, then switched to oral treatment, based on inflammatory index decrease and improvement of patient's clinical condition.

Only two $(2 \cdot 5 \%)$ children received a single antibiotic therapy; whereas the majority of the population received an association of antibiotics, as empiric choice. The most frequent antibiotic used was a third generation cephalosporin (ceftriaxone or cefotaxime) alone in one case, or associated only with metronidazole in six cases or with metronidazole plus another drug (such as vancomycin or teicoplanin) in nine subjects. In 18 patients, the empiric antibiotic regimen included a third generation cephalosporin in association with antibiotics other than metronidazole. Further antibiotics were used as initial treatment, in $29(36 \cdot 7 \%)$ cases.

Amoxicillin/clavulanate was primarily used, in the oral treatment.
Table 2. Location of solitary brain abscesses

\begin{tabular}{ll}
\hline \hline Site & No. of patients $(\%)$ \\
\hline Frontal lobe & $25(43 \cdot 1)$ \\
Parietal lobe & $13(22 \cdot 4)$ \\
Temporal lobe & $9(15 \cdot 5)$ \\
Occipital lobe & $5(8 \cdot 7)$ \\
Cerebellum & $4(6 \cdot 9)$ \\
Basal ganglia and thalamus & $2(3 \cdot 4)$ \\
\hline \hline
\end{tabular}

Antibiotic therapy was changed, according to microbiological results, as appropriate.

The mean length of overall antibiotic therapy was $59 \cdot 5 \pm 28 \cdot 4$ days (range 15-159 days); whereas the mean i.v. administration was $42 \cdot 8 \pm 18 \cdot 3$ days (range 5-120 days).

\section{Microbiology}

The pathogens responsible of the BA, isolated from blood, pus cultures or both, were documented in 43 $(54.4 \%)$ cases. The microorganisms isolated are shown in Table 3.

Analyzing separately children in whom BA was a consequence of spread of a contiguous infection, the most frequent responsible pathogens were Streptococcus spp. (11 cases) of which five were Streptococcus intermedius, isolated from pus. Three of them had a frontal localization of BA; whereas the other were one in temporal lobe and one in the occipital lobe. In four cases Staphylococcus spp. was isolated; four Enterobacteriaceae were isolated and two Anaerobes also. Lastly, 16 pus cultures were negative.

The Streptococcus spp. increased significantly through the study period, passing from three cases $(15 \cdot 7 \%)$ to 14 cases $(23.3 \%)$; whereas a reduction of Gram-negative bacteria was recorded.

\section{Outcomes}

Clinical sequelae were recorded in $31(39 \cdot 2 \%)$ children. Twenty-one of them had a single sequela: nine $(42.9 \%)$ epilepsy, five $(23.8 \%)$ focal motor deficits, three $(14 \cdot 3 \%)$ visual impairment, three (14.3\%) hydrocephalous, one $(4 \cdot 7 \%)$ language disease. Ten $(32 \cdot 2 \%)$ patients had multiple sequelae, of whom seven presented seizures associated to at least one other neurological sign (such as learning or behaviour disorders, language, visual or hearing disturbances), one had focal motor deficits and hearing diseases, one had hydrocephalous and behaviour disorders and the last 
Table 3. Type of pathogens isolated

\begin{tabular}{ll}
\hline \hline Type of microorganism & No. of cases \\
\hline Gram-positive cocci & \\
Viridans group Streptococci & $13^{\mathrm{a}}$ \\
Staphylococcus aureus & $4^{\mathrm{b}}$ \\
Coagulase negative Staphylococci & $4^{\mathrm{c}}$ \\
Streptococcus pneumoniae & $3^{\mathrm{d}}$ \\
B-Haemolytic Streptococci group B & 2 \\
Enterococcus faecalis & $1^{\mathrm{e}}$ \\
Enterobacteriaceae & \\
Enterobacter cloacae & $2^{\mathrm{f}}$ \\
Proteus mirabilis & 2 \\
Escherichia coli & $1^{\mathrm{g}}$ \\
Klebsiella oxytoca & 1 \\
Citrobacter koseri & $1^{\mathrm{h}}$ \\
Salmonella typhi & $1^{\mathrm{i}}$ \\
Anaerobes & 4 \\
Polimicrobial & 4 \\
Negative & 22 \\
\hline \hline
\end{tabular}

${ }^{\text {a }}$ One isolated from blood.

${ }^{\mathrm{b}}$ One isolated from cerebrospinal fluid (CSF).

${ }^{\mathrm{c}}$ One isolated from blood.

${ }^{\mathrm{d}}$ Two isolated from CSF and 1 from blood.

e Isolated from CSF.

${ }^{\mathrm{f}}$ Two isolated from blood.

${ }^{\mathrm{g}}$ Isolated from blood and 1 from CSF.

${ }^{\mathrm{h}}$ Isolated from blood.

${ }^{\mathrm{i}}$ Isolated from blood.

patient had focal motor deficits with learning disorders.

Clinical follow-up ranged from 1 to 109 months (median 6.4 months). In six (7.5\%) cases, a recurrent disease was reported. The mean duration of antibiotic therapy in children with a recurrent disease was not significantly different than those who have not $(64 \cdot 1$ vs. $59 \cdot 1$ days, respectively, $P=0 \cdot 78)$. The mortality rate was $1 \cdot 2 \%$ (one case). The death occurred after 2 months from the diagnosis.

At univariate analysis (shown on Table 4), the only two significant risk factors to develop clinical sequelae were the presence of neurological signs at admission $(P=0.003)$ and the duration of antibiotic treatment $(P=0.03)$.

\section{DISCUSSION}

This is the first multicentre Italian paediatric study which analyzes BA's incidence in our Country, delineating microbiological and clinical aspects and providing our experience on the management of this disease.

The gender and age distribution is similar to those reported by other recent studies [3,6], highlighting a male prevalence. In our study, the median age was 8.75 years, corresponding to the sinusitis, otitis media, mastoiditis and orbital cellulitis peak of incidence [11]. The number of BAs markedly increased during the two study periods (from 19 to 60 cases) probably provoked by the simultaneous increase, in Italy, of otitis media and mastoiditis' incidence $[12,13]$. In fact, the main predisposing factor, recorded in this survey, was a contiguous focus of infection, such as sinusitis, acute otitis or mastoiditis (16, 7 and 7 patients, respectively). Differently from other reports, the fungal BAs were excluded, so the immunocompromised population is scarce in this series [14].

In contrast to what reported by Manzar et al., we did not find congenital heart disease being a consistent predisposing condition for BA (six cases, 7.6\%), maybe because congenital heart diseases, in Italy, were early corrected [15]. In our analysis, immunosuppression and congenital heart diseases were not related to a major risk to develop a long-term sequela: this is probably due to a small sample size.

Clinical manifestations depend on the stage of the disease, virulence of the pathogen involved, immunological status of the patient, number, location of BA and presence or absence of associated meningitis or ventriculitis [5]. Most often the clinical symptoms are related to increase of intracranial pressure and are secondary to the focal effects of the mass and included non-specific manifestations, such as headache, nausea and vomiting [5]. In our series, as in other recent reports, the commonest symptoms at admission were non-specific, such as fever, headache and vomiting $[3,6,7]$. Instead some other authors found neurological signs as the major feature at the presentation, but, differently from our study, they have included headache in neurological symptoms and not in general ones [7,16].

The presentation with non-specific signs and symptoms could explain the delay in the diagnosis of BA; in our study, the median duration of symptoms before the diagnosis was 7 days. This is similar to those recorded in other European countries [2,3] and probably reflects a good clinical practice and an increasing diagnostic sensitivity towards this disease. Among neurological signs and symptoms seizures were the commonest, in line with Cole in her recent report [2].

Furthermore, in this survey, the presentation of a neurological sign or symptom on admission was statistically related to an increased risk to develop sequelae $(0=0.003)$. In our study, the more frequent 
Table 4. Results of univariate analysis for sequelae

\begin{tabular}{lllll}
\hline \hline Risk factor & $\begin{array}{l}\text { Patients with sequelae } \\
(n=31)\end{array}$ & $\begin{array}{l}\text { Patients without sequelae } \\
(n=48)\end{array}$ & OR $(95 \% \mathrm{CI})$ & $P$ \\
\hline Age $\leqslant 5$ years & $13(42)$ & $16(33 \cdot 3)$ & $1 \cdot 4(0 \cdot 5-3 \cdot 6)$ & $0 \cdot 59$ \\
Immunodeficiency & $0(0)$ & $4(8)$ & - & $0 \cdot 26$ \\
Congenital heart diseases & $2(6)$ & $4(8)$ & $0 \cdot 7(0 \cdot 1-4 \cdot 4)$ & $0 \cdot 89$ \\
Fever & $22(71)$ & $31(64 \cdot 5 \%)$ & $1 \cdot 3(0 \cdot 5-3 \cdot 5)$ & $0 \cdot 73$ \\
Neurological signs at admission & $18(58)$ & $11(23)$ & $4 \cdot 6(1 \cdot 7-12 \cdot 4)$ & $0 \cdot 003$ \\
Mean diameter, cm & $2 \cdot 88 \pm 1 \cdot 73$ & $2 \cdot 68 \pm 1 \cdot 44$ & - & $0 \cdot 57$ \\
Location & & & $0 \cdot 3(0 \cdot 1-1)$ & $0 \cdot 09$ \\
Frontal lobe & $7(22 \cdot 5)$ & $17(35 \cdot 4)$ & $2 \cdot 1(0 \cdot 6-7 \cdot 8)$ & $0 \cdot 32$ \\
Parietal lobe & $7(22 \cdot 5)$ & $5(10)$ & $0 \cdot 3(0-2 \cdot 8)$ & $0 \cdot 37$ \\
Occipital lobe & $1(3)$ & $4(8)$ & $2 \cdot 4(0 \cdot 5-11 \cdot 5)$ & $0 \cdot 27$ \\
Temporal lobe & $5(16)$ & $3(6)$ & $4 \cdot 3(0 \cdot 4-44)$ & $0 \cdot 30$ \\
Cerebellum & $3(9 \cdot 6)$ & $1(2)$ & $1 \cdot 3(0-22)$ & $0 \cdot 99$ \\
Other & $1(3)$ & $1(2)$ & $0 \cdot 4(0 \cdot 1-1 \cdot 5)$ & $0 \cdot 16$ \\
Pathogen & $7(22 \cdot 5)$ & $21(43)$ & $5(0 \cdot 9-25 \cdot 2)$ & $0 \cdot 08$ \\
$\quad$ Gram-positive cocci & $5(16)$ & $3(6)$ & $2 \cdot 3(0 \cdot 3-18 \cdot 5)$ & $0 \cdot 58$ \\
Enterobacteriaceae & $2(6)$ & $2(4)$ & $0 \cdot 9(0 \cdot 3-2 \cdot 5)$ & $0 \cdot 99$ \\
Anaerobes & $7(22 \cdot 5)$ & $12(25)$ & - & $0 \cdot 03$ \\
Antibiotic treatment only & $67 \cdot 8 \pm 33 \cdot 3$ & $53 \cdot 8 \pm 23 \cdot 3$ & & \\
Duration antibiotic treatment, days & & &
\end{tabular}

Values represent numbers of patients with percentages in parentheses or means \pm s.D., except where indicated otherwise.

neurological manifestations were generalized tonicclonic seizures, partial seizures and status epilepticus, often prolonged and severe at presentation, so longterm sequelae could be more possible in these patients.

Currently, lumbar puncture is not recommended because it can result in transforaminal or transtentorial herniation and consequent death $[10,17]$. Moreover, the microbiological analysis of cerebral fluid could not aid in the diagnosis of an unruptured BA. In our series, this procedure has been performed only in $31.6 \%$ of children, because of high suspicion of meningitis, and in all cases, there were no signs of increased intracranial pressure. The blood culture was positive only in 10 cases, underlying the need to use it during febrile episode, in order to document bacteraemia, thus directing the microbiological diagnosis.

The neuroimaging techniques, such as CT or MRI, allow to confirm BA diagnosis and to determine the location and the number of lesions [1]. CT imaging has proved to have many advantages: first, it can be already performed in the emergency department, allowing an immediate diagnosis. Second, it is useful in determining the location, size, mass effects, number of BA, together with providing information regarding the cause of BA, exploring also paranasal sinuses and mastoids [18,19]. Although MRI may be more sensitive in differentiating an abscess from other cystic lesions, the use of this technique is not recommended routinely in an acutely ill patient [18]. In our analysis, the vast majority of patients $(90 \cdot 7 \%)$ had CT scan on admission; MRI has been used in very few cases (seven, $9 \cdot 3 \%$ ) to diagnose BA.

As recently highlighted by other authors, even in our survey, the frontal lobe was the prevalent location $[6,7]$ : this is often provoked by the spread of a contiguous infection, especially from paranasal sinus, as shown in our analysis.

Gram-positive cocci, particularly viridans group streptococci, were the predominant pathogens isolated; consistent with other European series $[2,3,10]$. We have recorded a decline of Streptococcus pneumoniae in the pathogenesis of BAs, probably due to implementation of vaccine coverage of Italian paediatric population, with an estimated mean coverage in 2015 of $88 \cdot 73 \%$ [20]. In 22 cases, the pus culture was sterile: this could be explained by the previous administration of oral antibiotic before the diagnosis of BA, frequently happened in our setting $(45.6 \%$ of children) and also by the difficulty to obtain adequate sample for microbiological analysis.

Treatment of BAs is still controversial. GelabertGonzales et al. and Shachor-Meyouhas et al. had treated all their patients with a surgical approach 
[5,7]. Other authors, such as Cole, suggested a medical therapy alone only in selected cases [2]. In our series, the major part of children $(76 \%)$ underwent a surgical intervention. Children with stable neurological status, small BA's diameter and multiple abscesses, received only the medical treatment. Nowadays there are not clear indications to choose the medical approach alone instead of the surgical treatment. It is interesting that in our survey those who underwent also the surgical intervention were those who had a significant greater BA. Unfortunately, no correlations between site of BA and type of surgery emerged from our material, probably due to the high prevalence of frontal location in the whole study population, which might lead to a selection bias.

The choice, route and duration of empirical antibiotic regimen were extremely variable in our survey, reflecting the lack of consensus guideline. As suggested by other authors, an appropriate antimicrobial treatment should contemplate at least two agents: a third generation cephalosporin (ceftriaxone or cefotaxime) with metronidazole may be used to cover the most likely pathogens pending on specific organism identification [3-5, 21]. The boost with a carbapenem would potentially increase effective treatment because of its good spreading in cerebral tissue and its ability to counteract Gram-negative pathogens [3]. In case of high suspicion of methicillin-resistant Staphylococcus aureus infection, then vancomycin should be used [3]. According to more recent recommendations, the most frequent empiric antibiotic used, in our study, was a third generation cephalosporin (ceftriaxone or cefotaxime) alone or associated only with metronidazole or with metronidazole plus another drugs (such as vancomycin or teicoplanin).

Currently there are no national guidelines, in Italy, for the therapy of BA in paediatric setting, so also the duration of antimicrobial treatment, both i.v. and oral routes, is not yet standardized. In our series, the mean length of overall antibiotic therapy and the mean i.v. administration were similar to what reported by Felsenstein et al. and Gelabert-Gonzales et al. [3,5]. The duration of antibiotic treatment was significantly longer in patients who developed a sequela $(P=$ $0.032)$; this is because they were probably more severe cases.

The mortality is markedly declined in the last decades: we have reported a mortality rate of $1 \cdot 2 \%$, lower than the other European studies $[2,3,10]$. Clinical sequelae were recorded in $31(39 \cdot 2 \%)$ children, of which epilepsy and focal motor deficits were more frequent. In a small Spanish series GelabertGonzales et al. reported 4/28 children with motor deficit or visual impairment, four with hydrocephalous and 11 who needed an antiepileptic therapy [5]. Felsenstein et al. described an incidence of sequelae similar to us: $21 / 118$ patients with mild or moderate deficit and 12 with severe deficit [3].

In our survey, the recurrence rate was $7 \cdot 5 \%$, which is lower than in other studies [10]. The mean duration of antibiotic therapy was not different for patients with a recurrent disease; it is probably due to the small sample size.

The strength of this survey is the large number of Italian paediatric centres involved. Due to the retrospective nature of this study, some data were not available, especially concerning the follow-up of those diagnosed in the first period (1998-2000).

In conclusion, despite the estimated incidence of BA in developed countries is relatively low $(0 \cdot 9$ per 100000 person-years), clinicians should consider it when evaluating a child with seizures, headache or other neurologic symptoms, mainly if he had some predisposing factors [22].

The extreme variability in the antibiotic therapy, underlined by our material, focus the attention on the need to have standardized national guidelines or adequate recommendations on type and duration of antibiotic treatment.

\section{DECLARATION OF INTEREST}

The authors declare that they have no conflict of interest.

\section{REFERENCES}

1. Sheehan JP, et al. Brain abscess in children. Neurosurgical Focus 2008; 24: E6.

2. Cole TS, et al. Pediatric focal intracranial suppuration: a UK single-center experience. Child's Nervous System 2012; 28: 2109-2114.

3. Felsenstein $\mathbf{S}$, et al. Clinical and microbiologic features guiding treatment recommendations for brain abscesses in children. Pediatric Infectious Disease Journal 2013; 32: $129-135$.

4. Leotta $\mathbf{N}$, et al. Intracranial suppuration. Journal of Paediatrics and Child Health 2005; 41: 508-512.

5. Gelabert-González M, et al. Management of brain abscess in children. Journal of Paediatrics and Child Health 2008; 44: 731-735.

6. Ozsürekci Y, et al. Brain abscess in childhood: a 28-year experience. Turkish Journal of Pediatrics 2012; 54: 144-149. 
7. Shachor-Meyouhas Y, et al. Brain abscess in children epidemiology, predisposing factors and management in the modern medicine era. Acta Paediatrica 2010; 99: 1163-1167.

8. Brouwer MC, et al. Clinical characteristics and outcome of brain abscess: systematic review and meta-analysis. Neurology 2014; 82: 806-813.

9. Laulajainen-Hongisto A, et al. Intracranial abscesses over the last four decades; changes in aetiology, diagnostics, treatment and outcome. Infectious Diseases 2016; 48: 310-316.

10. Radoi M, Ciubotaru V, Tataranu L. Brain abscesses: clinical experience and outcome of 52 consecutive cases. Chirurgia 2013; 108: 215-225.

11. DeMuri GP, Wald ER. Complications of acute bacterial sinusitis in children. Pediatric Infectious Disease Journal 2011; 30: 701-702.

12. http://www.sipps.it/pdf/lineeguida/otite.pdf. Accessed 13th June 2017.

13. Marchisio P, et al. Diagnosis and management of acute mastoiditis in a cohort of Italian children. Expert Review of Anti Infective Therapy 2014; 12: 1541-1548.

14. Jansson AK, Enbland P, Sjolin J. Efficacy and safety of cefotaxime in combination with metronidazole for empirical treatment of brain abscess in clinical practice: a retrospective study of 66 consecutive cases. European
Journal of Clinical Microbiology and Infectious Diseases 2004; 23: 7-14.

15. Manzar N, et al. The study of the etiologic and demographic characteristics of intracranial brain abscess: a consecutive case series study from Pakistan. World Neurosurgery 2011; 76: 195-200.

16. Goodkin HP, Herper MB, Pomeroy SL. Intracerebral abscesses in children: historical trend at Children's Hospital in Boston. Pediatrics 2004; 113: 1765-1770.

17. Newcastle Upon Tyne Hospital NHS Foundation Trust. Antibiotic guidelines. In A\&E Handbook of Emergency Medicine. Newcastle Upon Tyne Hospital Trust NHS Foundation Trust, Newcastle, UK, 2009.

18. Moorthy RK, Rajshekhar V. Management of brain abscess: an overview. Neurosurgical Focus 2008; 24: E3.

19. Bernardini GL. Diagnosis and management of brain abscess and subdural empyema. Current Neurology and Neuroscience Reports 2004; 4: 448-456.

20. http://www.salute.gov.it/imgs/. Accessed on 13th March 2017.

21. Arlotti M, et al. Consensus document on controversial issues for the treatment of infections of the central nervous system: bacterial brain abscesses. International Journal of Infectious Diseases 2010; 14(Suppl. 4): S79-S92.

22. Calfee DP, Wispelwey B. Brain abscess. Seminars in Neurology 2000; 20: 353-360. 